\title{
Expression of the immune checkpoint molecule V-set immunoglobulin domain-containing 4 is associated with poor prognosis in patients with advanced gastric cancer
}

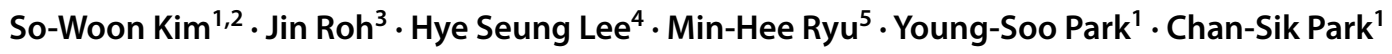

Received: 7 February 2020 / Accepted: 3 September 2020 / Published online: 14 September 2020

(c) The International Gastric Cancer Association and The Japanese Gastric Cancer Association 2020

\begin{abstract}
Background Recent clinical studies on immune checkpoint (IC) inhibitors in the context of advanced gastric cancer (AGC) have failed to show significant survival benefits but have suggested the possible role of IC inhibitors in anti-AGC immunity. Considering the low efficacy of targeted drugs in AGC, there is an urgent need for the discovery of new targets for the development of immunotherapeutics and prognostic markers for patient selection. This study aimed to investigate the expression of a new IC molecule, V-set Ig domain-containing 4 (VSIG4), and its clinical significance in AGC and other major cancers. Methods We analyzed the expression of VSIG4 and its correlation with survival in various carcinomas, including 882 surgically resected samples from patients with stage II-III AGC (two academic hospitals).

Results VSIG4 positivity in AGC was significantly associated with overall survival (OS; Hazard ratio (HR) $=2.661,95 \%$ confidence interval $[\mathrm{CI}]=2.012-3.519, P<0.001)$ and event-free survival $(\mathrm{HR}=2.8,95 \% \mathrm{CI}=2.18-3.72, P<0.001)$. These findings were successfully validated in independent cohorts. VSIG4 expression was also significantly correlated with low intratumoral CD8 + T-cell infiltration (CD8i) $(P=0.029)$ and high Foxp3 +/CD8i ratio $(P=0.026)$, which is consistent with the previously reported immunological function of VSIG4. However, VSIG4 expression was not associated with survival in other cancers (colon, $P=0.459$; lung, $P=0.275$; kidney, $P=0.121$; breast, $P=0.147$ ).

Conclusion Our results suggest that VSIG4 is an independent prognostic factor in AGC and also implies that VSIG4 is a second-tier IC molecule in AGC, thus, providing an important basis for the development of gastric cancer-specific immunotherapeutics.
\end{abstract}

Keywords V-set Ig domain-containing 4 - Immunotherapy · Advanced gastric cancer · Immune checkpoint molecule · Tumor microenvironment

Electronic supplementary material The online version of this article (https://doi.org/10.1007/s10120-020-01120-1) contains supplementary material, which is available to authorized users.

Young-Soo Park

youngsoopark@amc.seoul.kr

$\triangle$ Chan-Sik Park

csikpark@amc.seoul.kr

1 Department of Pathology, University of Ulsan College of Medicine, Asan Medical Center, Seoul, Republic of Korea

2 Department of Pathology, Kyung Hee University Hospital, Kyung Hee University College of Medicine, Seoul, Republic of Korea

\section{Introduction}

Gastric cancer is the third leading cause of cancer death worldwide [1]. For most patients with advanced gastric cancer, the benefit of chemotherapy beyond second-line therapies

3 Department of Pathology, Ajou University School of Medicine, Suwon, Republic of Korea

4 Department of Pathology, Seoul National University Bundang Hospital, Seongnam, Republic of Korea

5 Department of Oncology, University of Ulsan College of Medicine, Asan Medical Center, Seoul, Republic of Korea 
is marginal [2-8]. Moreover, most newly developed targeted therapies have failed in patients with advanced gastric cancer, and the survival rate is much lower than that in other cancers. Clinical studies on the use of immune checkpoint (IC) inhibitors for the treatment of advanced gastric cancer have shown both promise and limitations $[1,9,10]$. For example, in phase 3, ATTRACTION-2 study, nivolumab, an anti-programmed death (PD)-1 antibody, improved overall survival (OS) in patients with advanced gastric cancer when compared to a placebo [3]. Unlike conventional cytotoxic agents, nivolumab showed explicit long-term survival benefits and durable responses. In contrast KEYNOTE-061 trial, the use of another anti-PD-1 antibody, pembrolizumab, did not show any improvement in OS in the PD-L1-positive population ( $\geq 1 \%$ staining using the combined positive score [CPS], which is a proportional assessment of PD-L1 staining in both tumor and immune cells). However, pembrolizumab did have a pronounced benefit in patients who expressed high levels of PD-L1 (CPS $\geq 10$; hazard ratio $[\mathrm{HR}]=0.64,95 \%$ confidence interval $[\mathrm{CI}]=0.41-1.02$ ) [2]. These studies suggest that an anti-cancer immune reaction exists in patients with advanced gastric cancer, and it can be effective if correctly directed. Therefore, there is an urgent need to identify appropriate targets for immunotherapy as well as novel prognostic/predictive biomarkers for selecting patients who are more likely to benefit from particular therapies [11].

Recent studies have focused on new IC targets, including LAG-3, TIM-3, and TIGIT, which form the second tier of IC molecules with distinct, specific, and even organ-specific roles in immune response regulation [12, 13]. V-set Ig domain-containing 4 (VSIG4) is another second-tier IC molecule that belongs to the B7-related family [14]. VSIG4 is normally expressed on tissue-resident macrophages [14, 15] and functionally inhibits the production of IL-2, the proliferation of effector $\mathrm{CD} 8+\mathrm{T}$ cells, and induces regulatory $\mathrm{T}$ cells (Tregs) [16]. VSIG4 also inhibits the activation of proinflammatory macrophages by reprogramming mitochondrial pyruvate metabolism [17]. Recent studies have reported that VSIG4 expression in cancer cells was associated with poor prognosis in high-grade glioma and multiple myeloma [14, $18,19]$. Considering its potential target for immunotherapy, delineation of the prognostic value of VSIG4 expression in cancers in major organs is crucial. Therefore, we evaluated the expression of VSIG4 and its prognostic impact in advanced gastric cancer and other cancers. In addition, the correlation between VSIG4 expression and tumor-infiltrating inflammatory cells was analyzed.

\section{Materials and methods}

\section{Case selection}

Ethics approval for the study was obtained from the Institutional Review Board of Asan Medical Center, Seoul, Korea (project number 2015-0751), and the research was conducted in collaboration with Bundang Seoul National University Hospital in Bundang, Korea.

We retrospectively collected surgically resected and formalin-fixed paraffin-embedded (FFPE) specimens from advanced gastric cancer patients. All cases were reviewed to verify the diagnoses, and H\&E-stained sections were examined by two expert pathologists (S-W K and C-S P) to ensure that the tissues were representative of the diagnoses. A two-step, systematic review of the routine morphological and immunohistochemical aspects of the cases was carried out by authors who were blind to the diagnoses. Each slide was labeled with a unique study number. Tumors were diagnosed histologically based on WHO classification, and the pathological TNM stage was determined based on the guidelines established by the American Joint Committee on Cancer (AJCC) (8th edition)

[20]. Of the 1161 cases initially evaluated for the study, 279 cases were excluded based on the following exclusion criteria: treatment with neoadjuvant therapy, no clinical information available, and advanced gastric cancer stage I and IV at initial diagnosis. The final 882 cases included in the study were divided into discovery and validation sets of 485 and 397 cases, respectively. The discovery set consisted of 485 patients with advanced gastric cancer that underwent surgical resection in 2010, and the internal validation sets consisted of 193 patients who were treated at Asan Medical Center between 2005 and 2006. An independent external validation set was also obtained from patients treated at Bundang Seoul National University Hospital between 2003 and 2005. For tissue microarray (TMA) analyses, four cores were acquired from each case, two from the tumor invasion front (IF) and two from the tumor center (TC). Two expert pathologists (S-W K and C-S P) verified each diagnosis and representativeness of the cores for TMA by reviewing the tissue specimens. To minimalize the possible problem of intratumoral heterogeneity, two experts reviewed the HE slides and carefully selected the most representative areas morphologically. In addition, while reviewing the HE slides of all representative resection specimens, if there were differences in morphology such as tumor growth pattern or differentiation, the relevant areas were selected as cores together. Relevant demographic, clinical, and pathological data were recorded. Detailed characteristics of patients in the discovery and validation sets are summarized in Table 1 . 
Table 1 Demographic, clinical, and histological characteristics of 882 study patients with advanced gastric cancer

\begin{tabular}{|c|c|c|c|c|}
\hline \multirow[t]{2}{*}{ Parameter } & $\begin{array}{l}\text { All patients } \\
(N=882)\end{array}$ & $\begin{array}{l}\text { Discovery set } \\
(N=485)\end{array}$ & $\begin{array}{l}\text { Internal validation set } \\
(N=193)\end{array}$ & $\begin{array}{l}\text { External validation set } \\
(N=204)\end{array}$ \\
\hline & $N(\%)$ & $N(\%)$ & $N(\%)$ & $N(\%)$ \\
\hline \multicolumn{5}{|l|}{ Sex } \\
\hline Male & $598(67.8)$ & $335(69.1)$ & $128(66.3)$ & $135(66.2)$ \\
\hline Female & $284(32.2)$ & $150(30.9)$ & $65(33.7)$ & $69(33.8)$ \\
\hline \multicolumn{5}{|l|}{ Age, years } \\
\hline$<60$ & $368(41.7)$ & $244(50.3)$ & $35(18.1)$ & $89(43.6)$ \\
\hline$\geq 60$ & $514(58.3)$ & $241(49.7)$ & $158(81.9)$ & $115(56.4)$ \\
\hline Median (range) & $61(27-89)$ & $58(27-85)$ & $62(29-83)$ & $61(29-89)$ \\
\hline \multicolumn{5}{|l|}{ Tumor location } \\
\hline Upper & $238(27.0)$ & 105 (21.6) & $28(14.5)$ & $46(22.5)$ \\
\hline Middle & $183(20.7)$ & $101(20.8)$ & $36(18.7)$ & $45(22.1)$ \\
\hline Lower & $442(50.1)$ & $270(55.7)$ & $127(65.8)$ & $105(51.5)$ \\
\hline Entire & $19(2.2)$ & $9(1.9)$ & $2(1.0)$ & $8(3.9)$ \\
\hline \multicolumn{5}{|l|}{ Borrmann type } \\
\hline 1 & $22(2.5)$ & $12(2.5)$ & $1(0.5)$ & $5(2.5)$ \\
\hline 2 & $138(15.6)$ & $91(18.8)$ & $42(21.8)$ & $4(2.0)$ \\
\hline 3 & $489(55.4)$ & $322(66.4)$ & $139(72.0)$ & $138(67.6)$ \\
\hline 4 & $191(21.7)$ & $46(9.5)$ & $9(4.7)$ & $27(11.8)$ \\
\hline EGC-like & $33(3.7)$ & $12(2.5)$ & $0(0.0)$ & $24(11.8)$ \\
\hline Unclassified & $9(1.0)$ & $2(0.4)$ & $2(1.0)$ & $6(2.9)$ \\
\hline \multicolumn{5}{|l|}{ Lauren classification } \\
\hline Intestinal & $340(38.5)$ & $172(35.5)$ & $106(54.9)$ & $62(30.4)$ \\
\hline Diffuse & $399(45.2)$ & $227(46.8)$ & $54(28.0)$ & $118(57.8)$ \\
\hline Mixed & $143(16.3)$ & $86(17.8)$ & $33(17.1)$ & $24(11.8)$ \\
\hline \multicolumn{5}{|l|}{ Differentiation } \\
\hline WD & $16(1.8)$ & $9(1.9)$ & $4(2.1)$ & $3(1.5)$ \\
\hline MD & $275(31.2)$ & $127(26.6)$ & $82(42.5)$ & $66(32.4)$ \\
\hline PD & $399(45.2)$ & $181(37.9)$ & $90(46.6)$ & $103(50.5)$ \\
\hline PD with signet ring cell & $116(13.2)$ & $93(19.5)$ & $14(7.3)$ & $0(0.0)$ \\
\hline Signet ring cell & $54(6.1)$ & $22(4.6)$ & $3(1.6)$ & $23(11.3)$ \\
\hline Unclassified & $22(2.5)$ & $45(9.4)$ & $0(0.0)$ & $9(4.4)$ \\
\hline \multicolumn{5}{|l|}{ pStage (8th edition) } \\
\hline IIA & $286(27.7)$ & $110(22.9)$ & $12(6.2)$ & $38(16.3)$ \\
\hline IIB & $346(33.5)$ & $124(25.8)$ & $66(34.2)$ & $31(13.4)$ \\
\hline IIIA & $69(6.6)$ & $131(27.2)$ & $29(15.0)$ & $40(17.1)$ \\
\hline IIIB & $103(10.0)$ & $70(14.6)$ & $57(29.5)$ & $46(20.4)$ \\
\hline IIIC & $78(7.5)$ & $46(9.6)$ & $29(15.0)$ & $49(20.6)$ \\
\hline \multicolumn{5}{|l|}{ Adjuvant CTx } \\
\hline Not done & NA & $34(7.0)$ & NA & NA \\
\hline Done & NA & $451(93.0)$ & NA & NA \\
\hline \multicolumn{5}{|l|}{$\operatorname{MSI}(N=450)$} \\
\hline MSS/MSI-L & NA & 409 (90.9) & NA & NA \\
\hline MSI-H & NA & $41(9.1)$ & NA & NA \\
\hline \multicolumn{5}{|l|}{ Follow-up time, months } \\
\hline Median & 85.95 & 84 & 127 & 114.13 \\
\hline
\end{tabular}

$E G C$ early gastric cancer, $W D$ well differentiated, $M D$ moderately differentiated, $P D$ poorly differentiated, pStage pathological stage, CTx chemotherapy, NA not applicable, MSI microsatellite instability, MSS microsatellite stable, $M S I-L$ microsatellite instability-low, $M S I-H$ microsatellite instability-high 
To estimate OS and event-free survival (EFS), patients were followed-up from the date of surgery to the date of death or other events, such as metastasis or recurrence. The follow-up period was from January 2003 to August 2017. For a multi-organ comparison, 486 adenocarcinomas of the colon, 144 clear cell renal cell carcinomas, 144 invasive ductal carcinomas of the breast, and 251 nonsmall cell lung cancers were collected from the pathology archives at Asan Medical Center.

\section{Immunohistochemistry (IHC) and in situ hybridization (ISH)}

Staining was performed using an auto immunostainer (BenchMark XT; Ventana Medical Systems SA, Strasbourg, France) according to the manufacturer's instructions (Supplementary Materials). Each section was probed using an IHC panel designed to highlight tumor cells expressing VSIG4 (1:200, HPA003903; Sigma-Aldrich, St. Louis, MO, USA) and PD-L1 (1:100, E1L3N; Cell Signaling Technology, Danvers, MA, USA), and cell types in the infiltrates, which expressed CD8 (1:400, clone MONO; Dako, Carpinteria, CA, USA), FOXP3 (1:100, clone 236A/E7; Abcam, Cambridge, UK), CD68 (1:2000, KP-1; Dako), and CD163 (1:400, MOMO; Cell Marque, Rocklin, CA, USA). For advanced gastric cancer subgroupings, anti-P53 (1:1000, clone DO-7, Dako, Carpinteria, CA, USA) and E-cadherin (1:50, clone 4A2C7, Zymed, San Francisco, CA, USA) were also included in IHC. Specific binding of the anti-VSIG4 antibody was externally validated using the data hosted on the Human Protein Atlas Project database (https://www. proteinatlas.org/ENSG00000155659-VSIG4). Further, specific binding was also internally validated using a VSIG4transfected human embryonic kidney 293 T-cell line, as described in a previous report [19].

\section{Interpretation of the VSIG4 and PD-L1 results}

VSIG4 positivity in the TC and IF samples were defined as tumor cells showing cytoplasmic and/or membranous staining. There were 7 cases showing both cytoplasmic and nuclear staining. Nuclear positivity was not separately analyzed. Histoscore (H-score) was used for semi-quantitative analysis of VSIG4 immunoreactivity [21].The $\mathrm{H}$-score was calculated by the semi-quantitative valuation of staining intensity ( 0 , negative; 1 , weak; 2 , moderate; or 3 , strong) and by determining the percentage of stained tumor cells (Fig. 1). The H-scores ranged from 0 to 300 for each TMA core. Next, the average $\mathrm{H}$-score was calculated and used as representative values. VSIG4 expression in the tumor cells was classified as negative (VSIG4-) or positive (VSIG4+) based on the cutoff value determined using a web application for determination of optimal cutoff (Cutoff Finder; https:// molpath.charite.de/cutoff/) [22]. A cutoff score of 245 was best correlated with survival. When analyzing the expression of VSIG4, we checked the cases with marked discrepancy of Histoscore (H-score) between cores. Comparing VSIG4 positivity between each 4 cores from invasion front and tumor center using the cutoff of 245 , the representative average values from each invasion front and tumor center were dichotomized according to cutoff, and pairwise comparisons showed excellent concordance (97.9\%, Table 2). If there is discrepancy among cores, those cases were reviewed and double checked with anti-VSIG4 IHC on a whole section.

PD-L1 expression was evaluated in the tumor cells and tumor-infiltrating lymphocytes (TILs) of the TC and IF samples, as described previously [23]. PD-L1 staining was defined as membranous and/or cytoplasmic staining, and the staining intensity was graded on the following four point scale: 0 (negative), 1 (weak), 2 (moderate), or 3 (strong). The percentage of positive cells was measured in $1 \%$ increments. PD-L1-positive staining was defined as staining (weak to strong intensity) in more than $1 \%$ of cells. If the tumor cells or TILs were negative for PD-L1 staining in both the TC and IF samples, they were considered PD-L1 negative. All others (PD-L1-negative tumor cells and PD-L1-positive TILs, PDL1-positive tumor cells and PD-L1-negative TILs, and PDL1-positive tumor cells and TILs) were considered PD-L1 positive.

\section{Assessment of the tumor-associated immune contexture}

To evaluate the immune contexture, the TILs present in the tumor, including CD8- and Foxp3-positive T cells, and tumor-associated macrophages (TAMs), including CD68and CD163-positive cells, were analyzed by studying the IHC specimens. First, the tumor regions showing the highest density of immunoreactive cells were selected from IHC microphotographs scanned at low-power magnification. Then, within these regions, the absolute number of TILs (intraepithelial CD8-positive TILs [cytotoxic T cells, CD8i], and Foxp3-positive TILs [regulatory T cells (Tregs), Foxp3i]), and TAMs (stromal CD68-positive and CD163positive cells) was counted manually in a high-power field (HPF) view in the IF and TC of each tumor. The absolute number of immunoreactive TILs within a tumor cell cluster was counted. In cases of poorly cohesive carcinoma, only the TILs in direct contact with tumor cells were counted, and the average number of TILs in the core of each tumor was calculated. Subsequently, the ratios of the absolute numbers of immune cells (CD8i:Foxp3i and CD68:CD163) were calculated for each tumor. The total number of immune cells was determined as the sum of all evaluated cell types in the TC and IF, and the infiltration levels of the evaluated cell types were dichotomized (as high or low) for statistical analyses. 


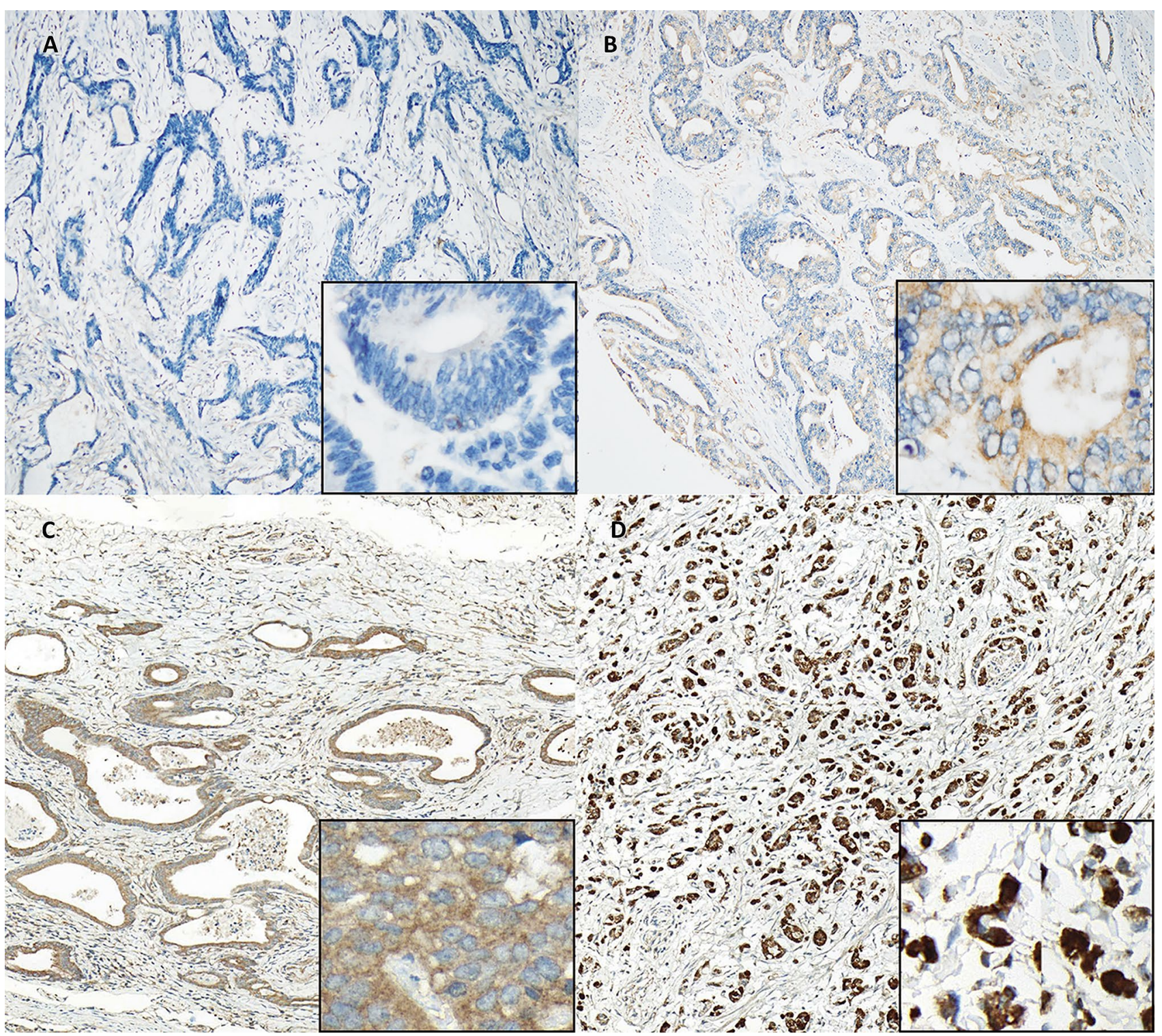

Fig. 1 Immunohistochemical staining for V-set Ig domain-containing 4 (VSIG4) expression in representative tumor samples. The tumor samples show the presence/absence of membranous/cytoplasmic VSIG4 staining of increasing intensity: a negative (0), b weak (1), c moderate (2), and d strong (3). Some cases with strong VSIG4 reactivity show nuclear staining as well. Original magnification, $40 \times$; Inset, $400 \times$. The presence of brown chromogen indicates VSIG4 staining. The blue color represents the hematoxylin counterstain
Table 2 Concordance rate of representative VSIG4 IHC value in invasion front and tumor center

\begin{tabular}{llllll}
\hline & VSIG4 & Tumor center & & $\begin{array}{l}\text { Total } \\
N\end{array}$ & $\begin{array}{l}\text { Con- } \\
\text { cordance } \\
\text { rate } \\
\%\end{array}$ \\
\cline { 3 - 6 } & & $\begin{array}{l}\text { Negative } \\
N(\%)\end{array}$ & $\begin{array}{l}\text { Positive } \\
N(\%)\end{array}$ & & 97.9 \\
\hline Invasion front & Negative, $N(\%)$ & $304(97.7)$ & $3(1.7)$ & 307 & \\
& Positive, $N(\%)$ & $7(2.3)$ & $171(98.3)$ & 178 & \\
& Total, $N$ & 311 & 174 & 485 & \\
\hline
\end{tabular}

IHC Immunohistochemical staining 
Each cell type in all tumor samples was classified as low or high based on the following optimal cutoff values, determined using Cutoff finder: CD8i high: > 10 CD8-positive T-cells/HPF, Foxp3i/CD8i high: Foxp3i/CD8i ratio $\geq 0.85$, CD68-high: > 110 CD68-positive macrophages, and CD163high: > 38 CD163-positive macrophages/HPF.

\section{Advanced gastric cancer subgroupings}

The study cohort was classified into five molecular subtypes based on the microsatellite instability (MSI) and EBV-status and the expression of E-cadherin and p53 proteins, as previously described [24]. Five subtypes were identified: cluster 1 (EBV-positive tumors), cluster 2 (MSI-High), cluster 3 (aberrant E-cadherin expression), cluster 4 (aberrant p53 expression), and cluster 5 (normal p53 expression) (Supplementary Materials).

\section{Digital image analysis}

Quantitative image analysis was used to reduce investigator-dependent errors. For quantitative analysis, digital slide images were obtained from the same IHC slides using the discovery set with a Pannoramic 250 Flash III (3DHistech, Hungary) and analyzed using CellProfiler v2.2.0 [25] (Supplementary Materials). VSIG4 expression was also found to be positive based on the staining intensity and proportion. The H-score was calculated, and a cutoff value of 175 was determined using Cutoff finder.

\section{Results}

\section{VSIG4 positivity was associated with poor OS and EFS}

The study aimed to assess VSIG4 expression in patients with advanced gastric cancer and evaluate its prognostic impact. In the discovery set, the median follow-up, as assessed by the Kaplan-Meier method, was 84 months, and 195 of the 485 patients $(40.2 \%)$ died during follow-up. One hundred seventy-seven of the 485 patients $(36.5 \%)$ were classified as VSIG4 +, and their median OS was 53.29 months versus 76.46 months for the 308 patients in the VSIG4 - group. Ten cases were showing discernable heterogeneity in $\mathrm{H}$-score among cores. Those cases were subject to confirming VSIG4 IHC on the whole section. The results of VSIG4 expression on whole sections were under those of corresponding TMA analysis. VSIG4 positivity in the discovery set was significantly associated with poor OS (Fig. $2 \mathrm{a}, P<0.001$, $\mathrm{HR}=2.661,95 \%$ confidence interval $[\mathrm{CI}]=2.012-3.519)$ when compared to the OS of VSIG4 - patients. The OS rate at 5 years was $78.4 \%$ in the VSIG 4 - group and $45.5 \%$ in the VSIG4 + group. Significantly more VSIG4 + patients had shorter EFS at 5 years than VSIG4- patients (Fig. 2b, $59.5 \%$ vs. $23.4 \%, P<0.001, \mathrm{HR}=2.8,95 \% \mathrm{CI}=2.18-3.72$ ). Validation was carried out in an independent non-overlapping cohort of 397 patients. Patients in the validation set were classified as VSIG4 + /- using the same cutoff score as that used in the discovery set. In the validation set, VSIG4 + was also significantly associated with poor OS and EFS (Fig. 2c and d, Internal validation set $[\mathrm{OS}] P=0.0065$, $\mathrm{HR}=2.10,95 \% \mathrm{CI}=1.49-2.96$; $[\mathrm{EFS}] P=0.006, \mathrm{HR}=2.03$, 95\% CI =1.45-2.76; Fig. 2e and f; External validation set [OS] $P=0.0013, \mathrm{HR}=1.59,95 \% \mathrm{CI}=1.11-2.27$; [EFS ] $P=0.0022, \mathrm{HR}=1.53,95 \% \mathrm{CI}=1.13-2.10)$. Quantitative image analysis was employed to reduce investigatordependent errors; it showed that high VSIG4 expression was associated with unfavorable outcomes (Fig. $2 \mathrm{~g}$ and h, [OS] $P<0.001, \mathrm{HR}=1.82,95 \% \mathrm{CI}=1.40-2.36$; [EFS $] P<0.001$, $\mathrm{HR}=1.34,95 \% \mathrm{CI}=1.12-2.15)$, which corroborates the results obtained using the manual analysis. Of note, a significant association between VSIG4 expression and patient survival was only observed in advanced gastric cancer.

VSIG4 expression in cancers of other organs, such as the colon, lung, breast, and kidney, showed no significant association with prognosis ( $[\mathrm{OS}]$ colon, $P=0.459$; lung, $P=0.275$; kidney, $P=0.121$; breast, $P=0.147$; Supplementary Fig. S1). In the cancers of kidney and breast, although statistically significant survival difference was not achieved between VSIG4-positive and negative groups, there were trends toward a poor overall survival in the VSIG4-positive group.

\section{VSIG4 positivity was associated with poor OS in subgroups delineated by molecular characteristics and stages}

The cohort was divided into the following five molecular subtypes based on a previous study [24]: subtype 1 (EBV positive, $N=13,2.7 \%$ ), subtype 2 (MSI-H, $N=40,8.2 \%$ ), subtype 3 (aberrant E-cadherin expression or EMT, $N=25$, $5.2 \%$ ), subtype 4 (aberrant P53 expression, $N=165,34 \%$ ), and subtype 5 (normal P53 expression, $N=207,42.7 \%$ ). Cases were included in the analysis only if their MSI status could be confirmed $(N=450)$. The correlation between OS and subtype was similar to that observed in previous reports [26], and MSI-H tumors showed the best prognosis, followed by tumors with normal P53 expression, tumors with aberrant P53 expression, and EMT tumors, which showed the worst prognosis $(P=0.053)$. Though the EBVpositive group showed the second-best prognosis in a previous report, in this study, the prognosis of this group was not distinct from that of the other groups. In all four subtypes, VSIG4 expression was related to significantly worse OS (Fig. 3a). Although the number of cases in the EBV-positive 
Fig. 2 Overall and event-free survival is strongly associated with VSIG4 expression. Kaplan-Meier curves of VSIG4-positive (VSIG4+) and VSIG4-negative (VSIG4-) patients with advanced gastric cancer belonging to different cohorts and subgroups; (a and b) discovery cohort; (c and d) internal validation cohort; (e and f) external validation cohort; and (g and $\mathbf{h}$ ) digital validation cohort. Symbols indicate censored observations, and colored sections indicate the confidence interval
Overall Survival
A. VSIG4 Discovery

$$
\text { -vsIG4- }+ \text { vsI64+ }
$$

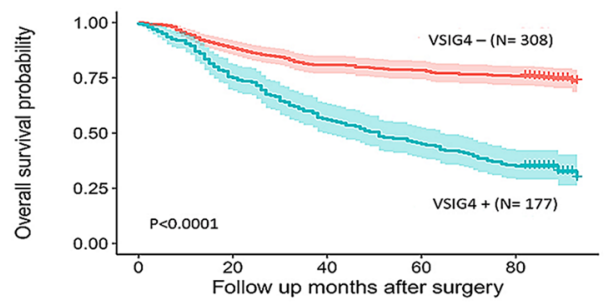

$\begin{array}{llccc}\text { VSIG4 }-308 & 260 & 237 & 232 & 195 \\ \text { VSIG4 + } 177 & 125 & 87 & 74 & 47\end{array}$

C. Internal Validation

$$
\mp \text { vsIG4- }+ \text { vSIG4 + }
$$

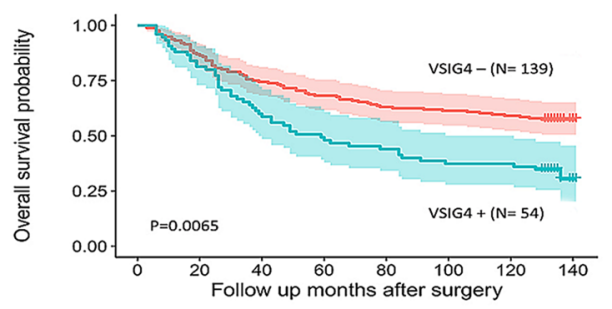

Number at Risk

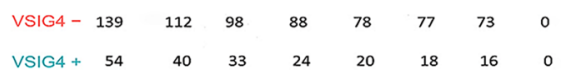

E. External Validation

$$
\text { + vsIG4 - + vsIG4 + }
$$

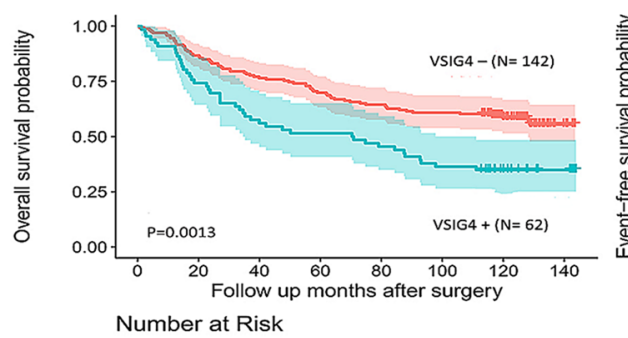
$\begin{array}{llllllll}\text { VSIG4 }-142 & 116 & 107 & 94 & 82 & 79 & 49 & 0 \\ \text { VSIG4 + } 62 & 42 & 35 & 30 & 26 & 20 & 13 & 0\end{array}$

G. Digital Validation

$$
\text { † vSIG4- }+ \text { vSIG4 + }
$$

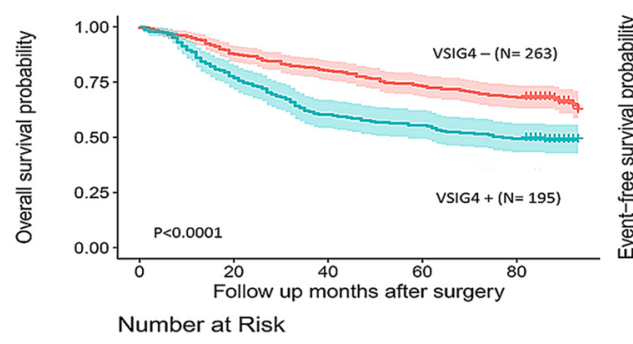

Number at Risk
Event-free Survival

B. VSIG4 Discovery

$$
\text { + vsIG4 - } \pm \text { vsIG4 + }
$$

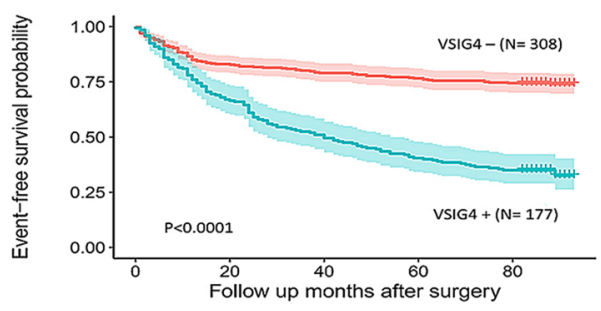

Number at Risk
D. Internal Validation

$$
\mp \text { vSIG4- }+ \text { vsIG4 + }
$$

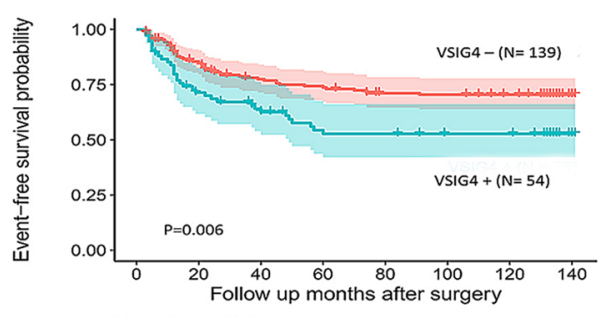
Number at Risk

$$
\begin{array}{llllllll}
\text { VSIG4 - } 139 & 101 & 91 & 85 & 76 & 75 & 72 & 0
\end{array}
$$

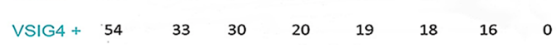

F. External Validation

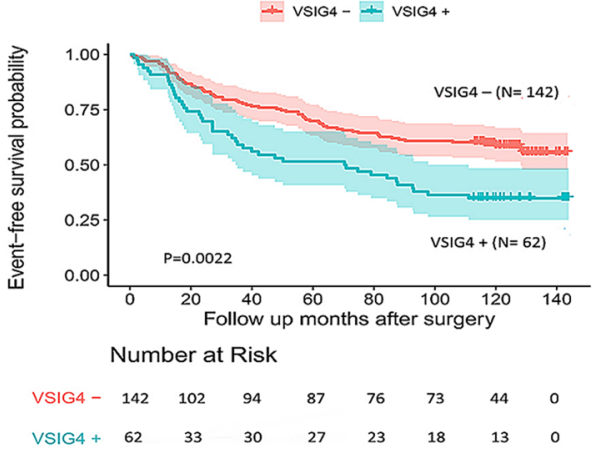

H. Digital Validation

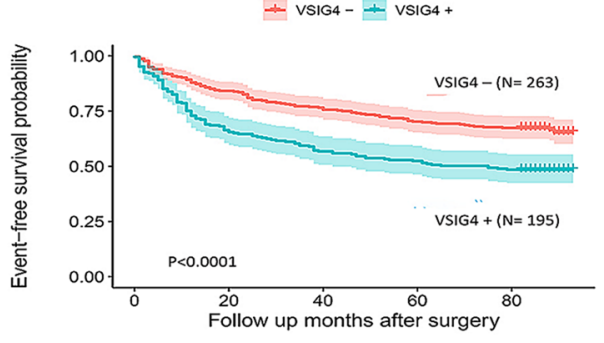
Number at Risk

$\begin{array}{lllll}\text { VSIG4 - } 263 & 216 & 201 & 182 & 147 \\ \text { VSIG4 + } 195 & 123 & 115 & 100 & 80\end{array}$




\section{A. Molecular grouping}
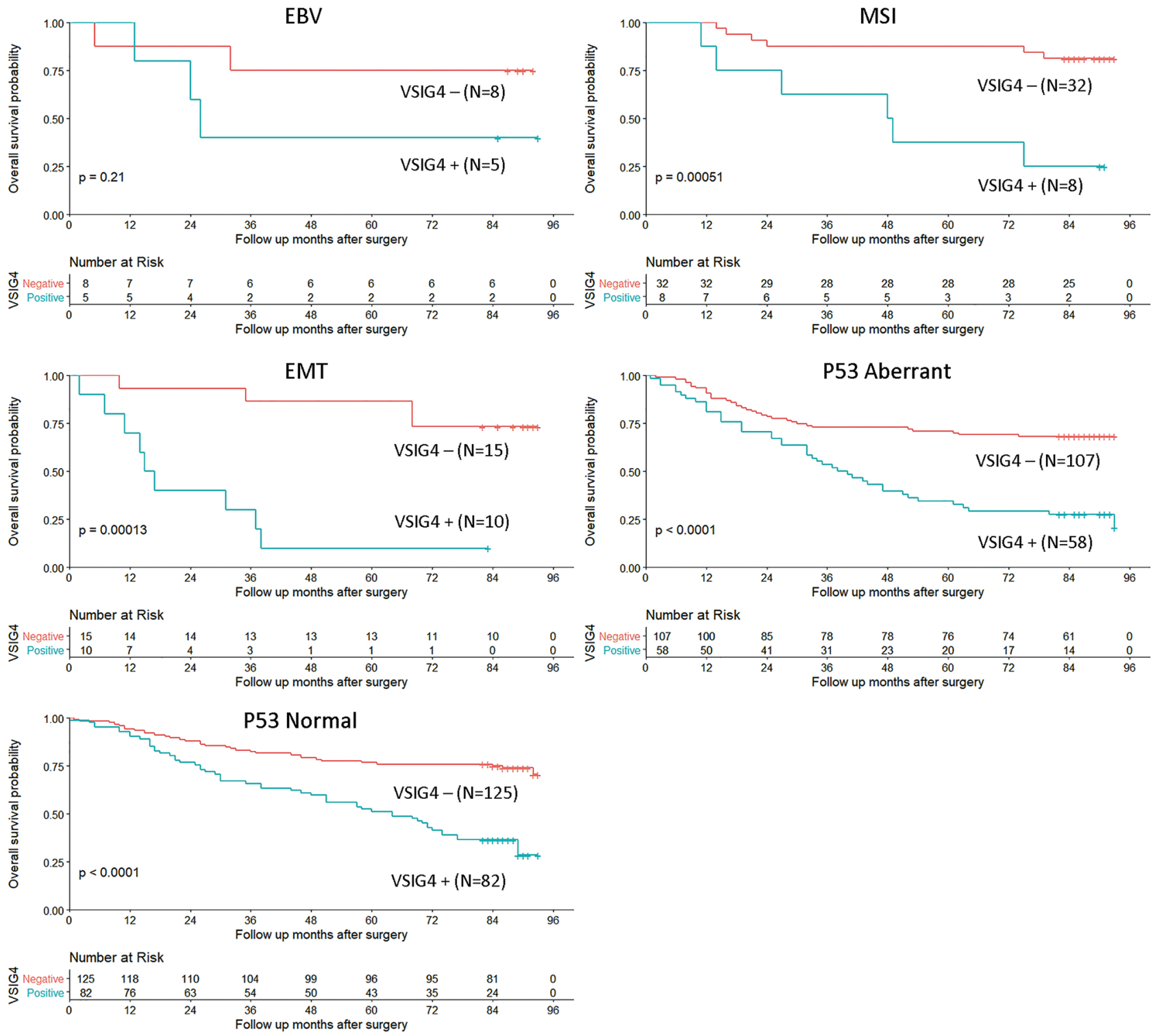

\section{B.Stage}

II
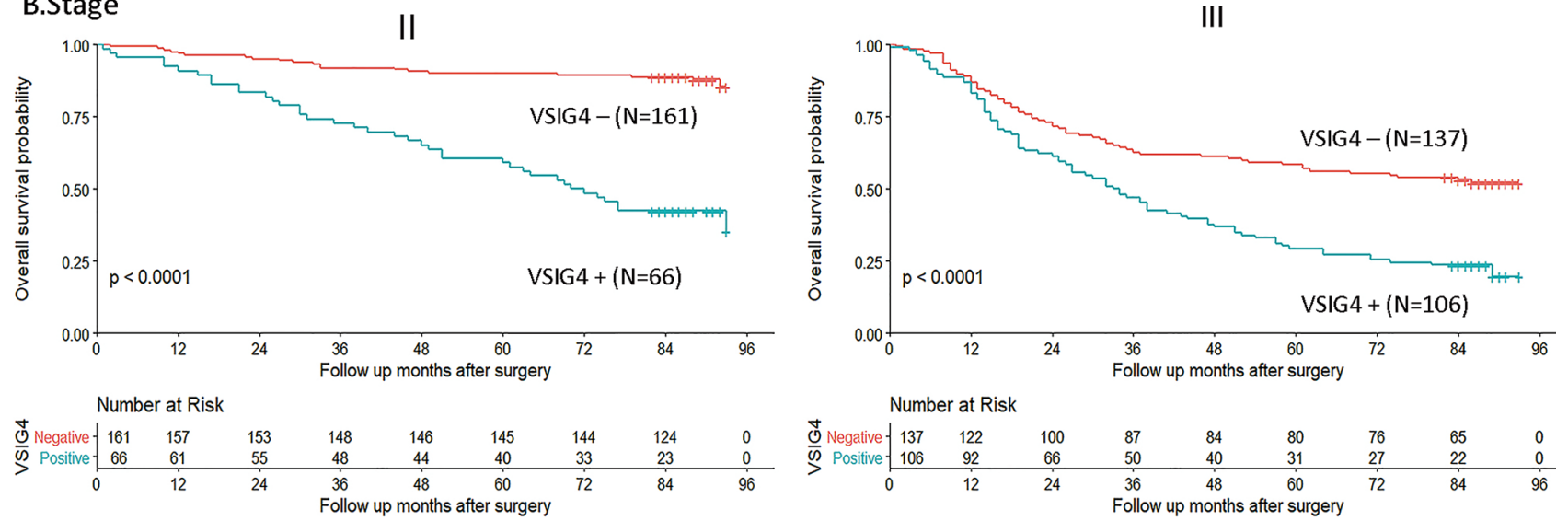
4Fig. 3 Kaplan-Meier plots of survival for patients with advanced gastric cancer based on molecular subgroup and stage and VSIG4 expression. a Subgrouping based on molecular expression reveals that VSIG4 expression is significantly associated with worse prognosis in all groups, except for the EBV group. For the EBV group, there is a trend toward poor prognosis, although it is not significant due to the small number of patients. b VSIG4 is a significant poor prognostic factor, even when stratified based on stage

group was small $(N=13)$, VSIG4 expression in the EBVpositive group showed a trend toward worse OS $(P=0.21)$. Together, the VSIG4 + group had a poor prognosis even when the patient group was divided into the pathological stage (pStage) (Fig. 3b).

\section{VSIG4 expression is correlated with low levels of CD8i infiltration and a high FoxP3i/CD8i ratio}

To address whether VSIG4 expression was associated with T-cell infiltration, we quantified the infiltrating immune cells in the tumor tissues of each patient by IHC and manually counted cells in scanned images. The absolute numbers of intraepithelial TILs were as follows: CD8i: median, 15 (range 0-233/HPF), and FoxP3i: median, 5 (range 0-66/ HPF). After dichotomization using cutoff values, the VSIG4 + group exhibited a significantly lower average density of CD8i cells than the VSIG4-group (Table 3; $P=0.029$ ), and the Foxp3i/CD8i ratio was significantly higher in the VSIG4 + group than that in the VSIG4- group $(P=0.026)$.

Survival analyses were conducted based on VSIG4 expression and CD8i density, and cases were placed in the following four subgroups: CD8i low/VSIG4-, CD8i low/ VSIG4 +, CD8i high/VSIG4 -, and CD8i high/VSIG4 + . The analyses showed that VSIG4 + patients had shorter OS and EFS, regardless of the CD8i density (high or low, $P<0.001)$. Interestingly, in the absence of VSIG4 expression the group with high CD8i density exhibited longer OS and EFS than the group with low CD8i density $(P=0.002)$, suggesting a possible role for CD8i in anti-tumor immunity. Moreover, for high CD8i density cases, OS was shorter in the VSIG4 + group (CD8i high/VSIG4 +) than that in the VSIG4 - group (CD8i high/VSIG4 -, $P<0.001$ ), suggesting a possible suppressive effect of VSIG4 on CD8i (Fig. 4a, b). Based on these results, we proposed that the poor prognostic impact of VSIG4 is related to anti-cancer immunity.

For tumor-associated macrophages, the density of CD68 expression was significantly different between the VSIG4 + and VSIG4 - groups $(P=0.012$, Supplementary Fig. S2). However, after stratifying the cases into CD68 low and high expression groups, no significant difference was observed between the two groups (Table $3, P=0.106$ ). There was no significant difference in CD163 expression and the CD163/CD68 ratio between the two groups (Table 3, $P=0.509$ ).

\section{VSIG4 expression is an independent prognostic factor}

Significant benefits concerning OS and EFS were observed in patients with gastric cancer in the VSIG4 - group who had a high CD8i density and a low FoxP3i/CD8i ratio. To determine whether VSIG4 expression or the density of immune cells was an independent prognostic factor, Cox regression analysis was performed for other clinicopathological factors (Table 4). Univariate analysis showed that VSIG4 expression, high Foxp3i/CD8i ratio, lymphovascular invasion (LVI), perineural invasion (PNI), and pStage were adverse prognostic factors for OS and EFS, while high CD8i, and MSI-H status were favorable prognostic factors for OS and EFS. Factors found to be significant in the univariate analysis were included in the multivariate analysis. After adjusting for these variables, VSIG4 expression, high CD8i, high Foxp3i/CD8i ratio, LVI, and pStage were found to be independent prognostic factors for OS and EFS (Fig. 4c). Notably, the HR of VSIG4 was markedly higher than that of pStage ([OS] VSIG4, HR=2.661, 95\% CI=2.012-3.519; pStage, $\mathrm{HR}=2.315,95 \% \mathrm{CI}=1.680-3.189)$.

\section{VSIG4 expression is not associated with histological subtypes}

Among total 882 cases, intestinal type, diffuse type, and mixed type were 340 (38.5\%), 399 (45.2\%), and 143 $(16.3 \%)$, respectively. There was no significant difference in VSIG4 expression among histological subtypes $(P=0.744$, Table 3). In addition, there was a significant association in VSIG4 expression and poor prognosis in each subtypes $(P<0.0001$, Supplementary Figure S3).

\section{VSIG4 expression and other clinicopathological characteristics}

A total of 882 patients were analyzed, and the detailed characteristics of the patients in the discovery and validation cohorts are summarized in Table 1. Briefly, the male to female ratio was $2.11: 1$, and the median age was 61 years (range 27-89 years). The median follow-up time was 85.95 months. VSIG4 expression was associated with LVI (60.5 versus $51.0 \%, P=0.047)$, and advanced pathological stage $(61.6 \%$ versus $45.5 \%, P=0.001)$. Of the analyzed cases, 40 of 450 (11.3\%) showed MSI-H, and this group showed significantly lower VSIG4 expression than the MSS group (4.9\% versus $11.1 \%, P=0.026)$. No significant differences in age, sex, tumor location, and Bormann type were noted between the VSIG4 + and VSIG4- groups. 
Table 3 Association of VSIG4 positivity with clinicopathological parameters in the discovery set of advanced gastric cancer $(N=485)$

\begin{tabular}{|c|c|c|c|c|c|c|c|}
\hline \multirow{2}{*}{\multicolumn{4}{|c|}{ parameters in the discovery set of advanced gastric cancer $(N=485)$}} & \multirow{4}{*}{ Parameter } & \multirow{4}{*}{$\begin{array}{l}\text { VSIG4 positive } \\
(N=177) \\
N(\%)\end{array}$} & \multirow{4}{*}{$\begin{array}{l}\text { VSIG4 negative } \\
(N=308) \\
N(\%)\end{array}$} & \multirow{4}{*}{$P$} \\
\hline & & & & & & & \\
\hline \multirow[t]{3}{*}{ Parameter } & \multirow{3}{*}{$\begin{array}{l}\text { VSIG4 positive } \\
(N=177) \\
N(\%)\end{array}$} & \multirow{3}{*}{$\begin{array}{l}\text { VSIG4 negative } \\
(N=308) \\
N(\%)\end{array}$} & \multirow[t]{3}{*}{$P$} & & & & \\
\hline & & & & & & & \\
\hline & & & & II & $29(16.4)$ & $62(20.1)$ & \\
\hline CD8i & & & $\mathbf{0 . 0 2 9}^{\mathrm{a}}$ & III & $115(64.0)$ & $207(67.2)$ & \\
\hline Low & $91(51.4)$ & $126(40.9)$ & & IV & $22(10.8)$ & $24(7.8)$ & \\
\hline High & $86(48.6)$ & $182(59.1)$ & & $\mathrm{V}$ & $5(0.5)$ & $7(2.3)$ & \\
\hline Foxp3i/CD8i ratio & & & $\mathbf{0 . 0 2 6}^{\mathrm{a}}$ & EGC-like & $1(4.4)$ & $1(0.3)$ & \\
\hline Low & $95(53.7)$ & $198(64.3)$ & & Lauren classification & & & 0.744 \\
\hline High & $82(46.3)$ & $110(35.7)$ & & Intestinal & $62(35.2)$ & $108(35.4)$ & \\
\hline CD68 & & & 0.106 & Diffuse & $82(46.6)$ & $143(46.9)$ & \\
\hline Low & $158(89.3)$ & $258(83.8)$ & & Mixed & $32(18.2)$ & $54(17.7)$ & \\
\hline High & $19(10.7)$ & $50(16.2)$ & & Differentiation & & & 0.505 \\
\hline CD163 & & & 0.509 & WD & $3(1.7)$ & $6(2.0)$ & \\
\hline Low & $88(49.7)$ & $164(53.2)$ & & MD & $51(29.1)$ & $76(25.2)$ & \\
\hline High & $89(50.3)$ & $144(46.8)$ & & $\mathrm{PD}$ & $59(33.7)$ & $122(40.4)$ & \\
\hline pStage (8th edition) & & & $0.001^{\mathrm{a}}$ & PD with signet ring cell & $32(18.3)$ & $61(20.2)$ & \\
\hline II & $68(38.4)$ & $168(54.5)$ & & Signet ring cell & $9(5.1)$ & $13(4.3)$ & \\
\hline III & 109 (61.6) & $140(45.5)$ & & Unclassified & $21(12.0)$ & $24(7.9)$ & \\
\hline
\end{tabular}

LVI

\section{Absent}

$70(39.5)$

Present

$107(60.5)$

PNI

Absent
Present
MSI $(N=450)$
MSS/MSL-L
MSI-H
Molecular subgroup
MSI-H $(N=40)$
EBV $(N=13)$
EMT $(N=25)$
P53-aberrant $(N=165)$
P53-normal $(N=207)$

$60(33.9)$

$117(66.1)$

$155(95.1)$

$8(4.9)$

$8(4.9)$

$5(3.1)$

$10(6.1)$

$58(35.6)$

$82(50.3)$

PD-L1

\section{Negative}

Positive

Age, years

$<60$

$\geq 60$

Median (range)

Sex

Male

Female

Location

Upper

Middle

Lower

Entire

$151(85.3)$

26 (14.7)

80 (45.2)

97 (54.8)

60 (27-83)

125 (70.6)

52 (29.4)

34 (19.2)

39 (22.0)

$101(57.1)$

$3(1.7)$

$151(49.0)$

157 (51.0)

113 (36.7)

$195(63.3)$

254 (88.5)

33 (11.5)

$32(11.1)$

8 (2.8)

15 (5.2)

107 (37.3)

125 (43.6)

$253(82.1)$

55 (17.9)

164 (53.2)

144 (46.8)

57 (31-85)

$210(68.2)$

98 (31.8)

71 (23.1)

$62(20.1)$

169 (54.9)

$6(2.1)$

Borrmann type

$$
\text { I }
$$$$
5(2.8)
$$

$\mathbf{0 . 0 2 1}^{\mathrm{a}}$

0.611

Table 3 (continued)

CD8i intraepithelial CD8 + T cell, Foxp3i intraepithelial FoxP3 + T cell, pStage pathological stage, LVI lymphovascular invasion, PNI perineural invasion, MSI microsatellite instability, MSS microsatellite stable, $M S I-L$ microsatellite instability-low, $M S I-H$ microsatellite instability-high, $W D$ well differentiated, $M D$ moderately differentiated, $P D$ poorly differentiated

$\mathbf{0 . 0 2 6}^{\text {a }} \quad{ }^{\text {a }}$ Significant, $P<0.05$

0.211

In addition, no significant between-group differences were noted for tumor differentiation and PNI. Comparison of the IF and TC showed that the intratumoral distribution of inflammatory infiltrates and immune modulator expression were homogeneous in most cases, indicating that the respective parameter categorizations for scoring groups (high versus low) were concordant in both tumor regions $(P=0.51)$. There is no association with VSIG4 expression and molecular subtypes by the Chi-square test for the categorical variable $(P=0.211)$ and the Kruskal-Wallis test for the continuous variable $(P=0.06)$. For PD-L1, there was no significant difference in survival between groups with high and low VSIG4 expression $(P=0.380)$. Other clinicopathological characteristics are summarized in Table 3.

\section{Discussion}

0.777

There are several obstacles to the development of effective therapies for advanced gastric cancer [27]. First, gastric cancer is a heterogeneous malignancy with diverse histological types and genotypes. Second, no prognostic/ predictive biomarkers have been established for the selection of optimal therapies for patients with advanced gastric 

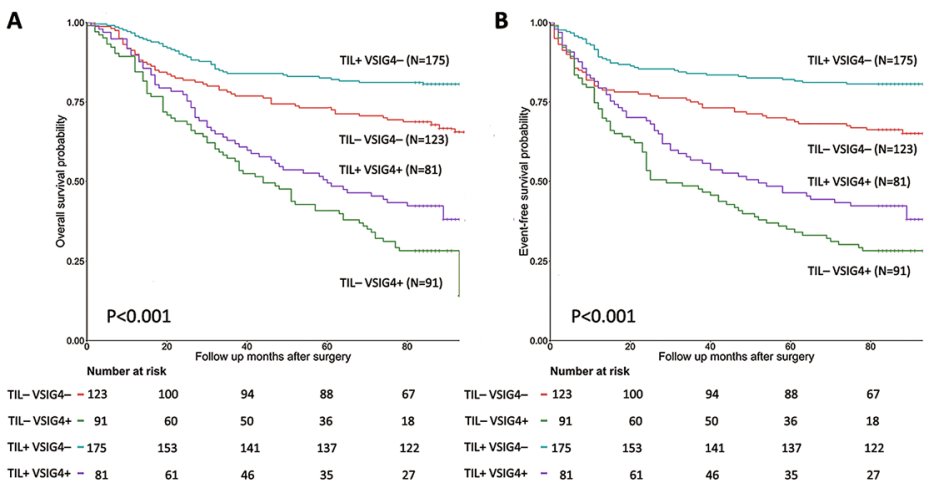

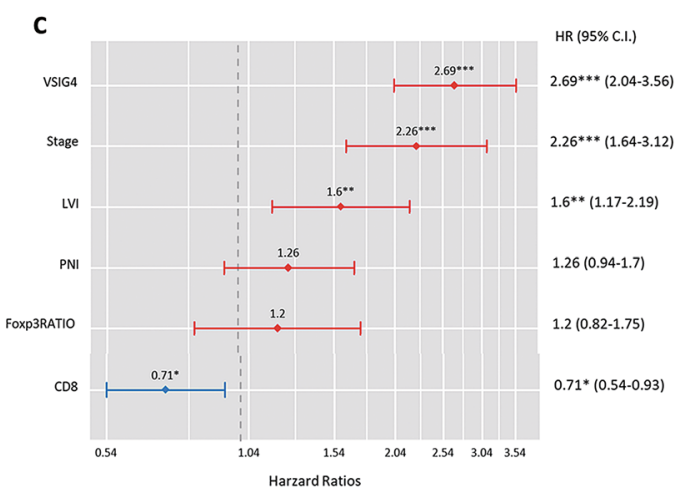

Fig. 4 Kaplan-Meier curves of (a) overall survival (OS) and (b) event-free survival (EFS) for patients with advanced gastric cancer stratified according to VSIG4 expression and intraepithelial CD8positive T-cell (CD8i) density. The group with high CD8i density has a longer OS and EFS than the group with low CD8i density in the absence of VSIG4 expression. Panel c shows the hazard ratio (HR) and $95 \%$ confidence intervals (CI) for OS in the biomarker subgroups.
VSIG4 expression is an independent poor prognostic factor for OS. Stratified HRs are given for VSIG4, PD-L1, CD8i, intraepithelial Foxp3 + T cell/CD8 ratio (Foxp3i/CD8i ratio), lymphovascular invasion (LVI), and pathologic stage (pStage). HRs and 95\% CIs were computed using the Cox proportional-hazards model, and the $P$ values were obtained from a stratified log-rank test
Table 4 Overall survival analyses of patients with advanced gastric cancer (discovery set, $N=485$ )

\begin{tabular}{|c|c|c|c|c|c|c|}
\hline \multirow[t]{2}{*}{ Factor } & \multicolumn{2}{|c|}{ Univariate analysis } & \multirow[t]{2}{*}{$P$} & \multicolumn{2}{|c|}{ Multivariate analysis } & \multirow[t]{2}{*}{$P$} \\
\hline & $\overline{\mathrm{HR}}$ & $95 \% \mathrm{CI}$ & & $\overline{\mathrm{HR}}$ & $95 \% \mathrm{CI}$ & \\
\hline VSIG4 & & & $<\mathbf{0 . 0 0 1}^{\mathrm{a}}$ & & & $<\mathbf{0 . 0 0 1}^{\mathrm{a}}$ \\
\hline Negative & 1 & & & 1 & & \\
\hline Positive & 3.248 & $2.467-4.278$ & & 2.661 & $2.012-3.519$ & \\
\hline pStage (8th edition) & & & $<\mathbf{0 . 0 0 1}^{\mathrm{a}}$ & & & $<\mathbf{0 . 0 0 1}^{\mathrm{a}}$ \\
\hline II & 1 & & & 1 & & \\
\hline III & 3.134 & $2.328-4.219$ & & 2.315 & $1.680-3.189$ & \\
\hline PD-L1 & & & 0.054 & & & \\
\hline Negative & 1 & & & & & \\
\hline Positive & 0.672 & $0.449-1.006$ & & & & \\
\hline $\mathrm{CD} 8 \mathrm{i}$ & & & $0.002^{\mathrm{a}}$ & & & $\mathbf{0 . 0 1 3}^{\mathrm{a}}$ \\
\hline Low & 1 & & & 1 & & \\
\hline High & 0.647 & $0.494-0.847$ & & 0.708 & $0.540-0.929$ & \\
\hline Foxp3i/CD8i ratio & & & $\mathbf{0 . 0 0 4}^{\mathrm{a}}$ & & & $\mathbf{0 . 0 2 3}^{\mathrm{a}}$ \\
\hline Low & 1 & & & 1 & & \\
\hline High & 1.480 & $1.130-1.939$ & & 1.02 & $0.71-1.46$ & \\
\hline LVI & & & $<0.001^{\mathrm{a}}$ & & & $\mathbf{0 . 0 0 6}^{\mathrm{a}}$ \\
\hline Absent & 1 & & & 1 & & \\
\hline Present & 2.383 & $1.778-3.193$ & & 1.553 & $1.134-2.126$ & \\
\hline PNI & & & $0.021^{\mathrm{a}}$ & & & 0.107 \\
\hline Absent & 1 & & & 1 & & \\
\hline Present & 1.415 & $1.054-1.899$ & & 1.276 & $0.948-1.716$ & \\
\hline
\end{tabular}

$C I$ confidence interval, $C D 8 i$ intraepithelial CD8 + T cell, FoxP3i intraepithelial Foxp3 + T cell, $L V I$ lymphovascular invasion, $P N I$ perineural invasion, $p$ Stage pathological Stage

${ }^{\text {a }}$ Significant, $P<0.05$ cancer. Therefore, the identification of reliable biomarkers could lead to better outcomes in advanced gastric cancer. This study is the first to evaluate VSIG4 expression in large cohorts in major human cancers. We found that VSIG4 expression in advanced gastric cancer is strongly associated with poor OS and EFS. It was a significant independent prognostic factor in the multivariate analysis, and its importance was supported by both internal and external validation 
as well as by separate objective digital methods. This result is in agreement with previous reports that demonstrated associations between VSIG4 expression and poor prognosis in other cancers. Notably, the HR of VSIG4 expression for OS and EFS was remarkably higher than that for PD-L1 and pStage, suggesting the potential of VSIG4 as a crucial prognostic marker for advanced gastric cancer.

TILs contribute to the immune response against tumors, and many studies have reported an association between TILs and clinical outcomes in patients with various types of cancer [28-30]. Regarding the immune contextures in GC, the current study showed that high levels of TILs were distinctly associated with better OS in agreement with previous reports [24, 28-31]. Interestingly, VSIG4 positivity was associated with a high ratio of tumor-infiltrating Foxp3 + Tregs/CD8i cells and a low level of CD8i T-cell infiltration. Besides, among cases with high CD8i density, OS was worse in the VSIG4 + group than that in the VSIG4 - group, suggesting a probable suppressive role for VSIG4 against CD8i. These results are consistent with previous immunological studies that reported that VSIG4 suppresses T-cell proliferation by attenuating the activation of multiple T-cell receptor signaling cascades [32] and that VSIG4 can promote Treg differentiation and increase the stability of Tregs [15]. Therefore, it is plausible that the effect of VSIG4 in advanced gastric cancer may be related to anti-tumor immunity, suggesting possible suppressive and inductive roles of VSIG4 in CD8i cells and Tregs, respectively.

In the recent ATTRACTION-2 phase 3 study, IC inhibitors were shown to be useful for gastric cancer; this was also supported by many PD-1/PD-L1 studies. However, their role as practical prognostic/predictive markers is controversial, and current immunotherapeutic strategies remain meager [1, 10, 26, 33-37]. Therefore, an appropriate selection of patients who may potentially benefit from given immunotherapy is essential, and a consensus must be reached regarding the IHC-based determination of IC molecules and TIL levels.

There was a significant negative correlation between VSIG4 expression and survival in advanced gastric cancer. However, for other solid tumors, VSIG4 expression was not associated with survival. In some tumors, high VSIG4 expression showed a tendency toward shorter survival, but this was not significant. Many recent studies have highlighted the importance of alternative checkpoint inhibitors as emerging targets for future mono/combination therapies [38]. Cytotoxic T-lymphocyte-associated antigen-4 (CTLA4) and PD-1 checkpoint inhibitors are being investigated for their immunotherapy potential and other checkpoints with potentially greater potency show promise for improving patient outcomes. For example, Anderson et al. postulated that CTLA- 4 and PD- 1 could serve as "first tier" co-target receptors to sustain overall immune self-tolerance. In contrast, other receptors, such as TIGIT, LAG-3, and TIM-3, could serve as "second-tier" targets, as they exhibit distinct functions, especially in tissues where they regulate distinct aspects of immunity [12]. These "second-tier" co-inhibitory receptor pathways may dominate in sites where their ligands and/or the receptors themselves are highly expressed. Considering the clinical impact of VSIG4 expression mainly in advanced gastric cancer, VSIG4 may be a "second-tier" immune checkpoint molecule in the gastric immune microenvironment. Increased understanding of the specialized function of VSIG4 will aid the rational development and application of therapies that target VSIG4 in advanced gastric cancer. Our study is limited by its retrospective nature and by the possible incomplete representation of the TMA of the cancer tissue due to heterogeneity of AGC. Despite the limitations, if interpreted carefully, it might serve as an important basis for future studies investigating the role of VSIG4 in immune microenvironment and the development of new therapy for patients with AGC. More correlative studies are warranted to strengthen this finding.

In conclusion, our results indicate that VSIG4 overexpression in advanced gastric cancer is independently and strongly related to poor prognosis. Thus, VSIG4 has the potential for use as a prognostic marker, and it can serve as a promising target molecule for novel immunotherapies against advanced gastric cancer.

Acknowledgements We acknowledge and thank the following people who have helped: Jiwon Jung and Junho Lee

Author contributions Conception and design: S-WK, MR, YSP, and C-SP. Development of methodology: IC, MR, and YSP. Acquisition of data (provided animals, acquired and managed patients, provided facilities, etc.): S-WK, C-SP, JR, JK, HG, HL, A-NL, YC, and SJ. Analysis and interpretation of data (e.g., statistical analysis, biostatistics, computational analysis): S-WK, C-SP, and JR. Writing, review, and/or revision of the manuscript: S-WK, C-SP, and JR. Administrative, technical, or material support (i.e., reporting or organizing data, constructing databases): JK, HG, and HL.

Funding This study was supported by grants from the Asan Institute for Life Sciences, Asan Medical Center, Seoul, Korea (Grant No. 2019IL0841 to Y-S P and Grant No. 2019IL0527 to C-S P) and the National Research Foundation of Korea (NRF) funded by the Ministry of Science, ICT and Future Planning (NRF-2020R1A2C2006362 to C-S P).

Data availability Not applicable.

\section{Compliance with ethical standards}

Competing interests The authors declare no potential conflicts of interest.

Ethics approval and consent to participate The study protocol was approved by the Institutional Review Board of Asan Medical Center, Seoul, Korea (project number 2015-0751). 
Consent for publication Not applicable.

\section{References}

1. Shitara K, Ozguroglu M, Bang YJ, et al. Pembrolizumab versus paclitaxel for previously treated, advanced gastric or gastrooesophageal junction cancer (KEYNOTE-061): a randomised, open-label, controlled, phase 3 trial. Lancet. 2018;392:123-33. https://doi.org/10.1016/S0140-6736(18)31257-1.

2. Fuchs CS, Doi T, Jang RW, et al. Safety and efficacy of pembrolizumab monotherapy in patients with previously treated advanced gastric and gastroesophageal junction cancer: phase 2 clinical KEYNOTE-059 trial. JAMA Oncol. 2018;4:e180013. https://doi. org/10.1001/jamaoncol.2018.0013.

3. Bang YJ, Van Cutsem E, Feyereislova A, et al. Trastuzumab in combination with chemotherapy versus chemotherapy alone for treatment of HER2-positive advanced gastric or gastrooesophageal junction cancer (ToGA): a phase 3, open-label, randomised controlled trial. Lancet. 2010;376:687-97. https://doi. org/10.1016/S0140-6736(10)61121-X.

4. Li J, Qin S, Xu J, et al. Randomized, double-blind, placebo-controlled phase III trial of apatinib in patients with chemotherapyrefractory advanced or metastatic adenocarcinoma of the stomach or gastroesophageal junction. J Clin Oncol. 2016;34:1448-544. https://doi.org/10.1200/JCO.2015.63.5995.

5. Johnson DB, Frampton GM, Rioth MJ, et al. Targeted next generation sequencing identifies markers of response to PD-1 blockade. Cancer Immunol Res. 2016;4:959-67. https://doi. org/10.1158/2326-6066.CIR-16-0143.

6. Kang YK, Boku N, Satoh T, et al. Nivolumab in patients with advanced gastric or gastro-oesophageal junction cancer refractory to, or intolerant of, at least two previous chemotherapy regimens (ONO-4538-12, ATTRACTION-2): a randomised, double-blind, placebo-controlled, phase 3 trial. Lancet. 2017;390:2461-71. https ://doi.org/10.1016/S0140-6736(17)31827-5.

7. Salati M, Di Emidio K, Tarantino V, et al. Second-line treatments: moving towards an opportunity to improve survival in advanced gastric cancer? ESMO Open. 2017;2:e000206. https:// doi.org/10.1136/esmoopen-2017-000206.

8. Peeters M, Cervantes A, Moreno Vera S, et al. Trifluridine/tipiracil: an emerging strategy for the management of gastrointestinal cancers. Future Oncol. 2018;14:1629-45. https://doi.org/10.2217/ fon-2018-0147.

9. Fuchs CS, Tomasek J, Yong CJ, et al. Ramucirumab monotherapy for previously treated advanced gastric or gastro-oesophageal junction adenocarcinoma (REGARD): an international, randomised, multicentre, placebo-controlled, phase 3 trial. Lancet. 2014;383:31-9. https://doi.org/10.1016/S0140-6736(13)61719-5.

10. Wilke H, Muro K, Van Cutsem E, et al. Ramucirumab plus paclitaxel versus placebo plus paclitaxel in patients with previously treated advanced gastric or gastro-oesophageal junction adenocarcinoma (RAINBOW): a double-blind, randomised phase 3 trial. Lancet Oncol. 2014;15:1224-355. https://doi.org/10.1016/S1470 -2045(14)70420-6.

11. Gide TN, Quek C, Menzies AM, et al. Distinct immune cell populations define response to anti-PD-1 monotherapy and anti-PD-1/ anti-CTLA-4 combined therapy. Cancer Cell. 2019;35(23855):e6. https://doi.org/10.1016/j.ccell.2019.01.003.

12. Anderson AC, Joller N, Kuchroo VK. Lag-3, Tim-3, and TIGIT: co-inhibitory receptors with specialized functions in immune regulation. Immunity. 2016;44:989-1004. https://doi.org/10.1016/j. immuni.2016.05.001.
13. Shen P, Yue R, Tang J, et al. Preferential Tim-3 expression on Treg and CD8(+) T cells, supported by tumor-associated macrophages, is associated with worse prognosis in gastric cancer. Am J Transl Res. 2016;8:3419-28.

14. Liao Y, Guo S, Chen Y, et al. VSIG4 expression on macrophages facilitates lung cancer development. Lab Invest. 2014;94:70615. https://doi.org/10.1038/labinvest.2014.73.

15. Yuan X, Yang BH, Dong Y, et al. CRIg, a tissue-resident macrophage specific immune checkpoint molecule, promotes immunological tolerance in NOD mice, via a dual role in effector and regulatory T cells. eLife. 2017. https://doi.org/10.7554/eLife .29540 .

16. Vogt L, Schmitz N, Kurrer MO, et al. VSIG4, a B7 familyrelated protein, is a negative regulator of $\mathrm{T}$ cell activation. J Clin Invest. 2006;116:2817-26. https://doi.org/10.1172/JCI25673.

17. Li J, Diao B, Guo S, et al. VSIG4 inhibits proinflammatory macrophage activation by reprogramming mitochondrial pyruvate metabolism. Nat Commun. 2017;8:1322. https://doi. org/10.1038/s41467-017-01327-4.

18. Xu T, Jiang Y, Yan Y, et al. VSIG4 is highly expressed and correlated with poor prognosis of high-grade glioma patients. Am J Transl Res. 2015;7:1172-80.

19. Roh J, Jeon Y, Lee AN, et al. The immune checkpoint molecule $\mathrm{V}$-set Ig domain-containing 4 is an independent prognostic factor for multiple myeloma. Oncotarget. 2017;8:58122-32. https ://doi.org/10.18632/oncotarget.19468.

20. Amin MB, Edge SB, Greene FL. AJCC cancer staging manual. 8th ed. Chicago: Springer; 2017.

21. Hirsch FR, Varella-Garcia M, Bunn PA Jr, et al. Epidermal growth factor receptor in non-small-cell lung carcinomas: correlation between gene copy number and protein expression and impact on prognosis. J Clin Oncol. 2003;21:3798-807. https:// doi.org/10.1200/JCO.2003.11.069.

22. Budczies J, Klauschen F, Sinn BV, et al. Cutoff Finder: a comprehensive and straightforward Web application enabling rapid biomarker cutoff optimization. PLoS ONE. 2012;7:e51862. https://doi.org/10.1371/journal.pone.0051862.

23. Boger C, Behrens HM, Mathiak M, et al. PD-L1 is an independent prognostic predictor in gastric cancer of Western patients. Oncotarget. 2016;7:24269-83. https://doi.org/10.18632/oncot arget.8169.

24. Kim JY, Kim WG, Kwon CH, et al. Differences in immune contextures among different molecular subtypes of gastric cancer and their prognostic impact. Gastric Cancer. 2019;22:1164-75. https://doi.org/10.1007/s10120-019-00974-4.

25. Carpenter AE, Jones TR, Lamprecht MR, et al. Cell Profiler: image analysis software for identifying and quantifying cell phenotypes. Genome Biol. 2006;7:R100. https://doi.org/10.1186/ gb-2006-7-10-r100.

26. Cancer Genome Atlas Research Network. Comprehensive molecular characterization of gastric adenocarcinoma. Nature. 2014;513:202-9. https://doi.org/10.1038/nature13480.

27. Nagai $H$, Muto M. Optimal management of immune-related adverse events resulting from treatment with immune checkpoint inhibitors: a review and update. Int J Clin Oncol. 2018;23:410-20. https://doi.org/10.1007/s10147-018-1259-6.

28. Ropponen KM, Eskelinen MJ, Lipponen PK, et al. Prognostic value of tumour-infiltrating lymphocytes (TILs) in colorectal cancer. J Pathol. 1997;182:318-24. https://doi. org/10.1002/(SICI)1096-9896(199707)182:3<318:AID-PATH8 $62>3.0 . \mathrm{CO} ; 2-6$.

29. Lipponen PK, Eskelinen MJ, Jauhiainen K, et al. Tumour infiltrating lymphocytes as an independent prognostic factor in transitional cell bladder cancer. Eur J Cancer. 1992;29A:69-75. https://doi.org/10.1016/0959-8049(93)90579-5. 
30. Karja V, Aaltomaa S, Lipponen P, et al. Tumour-infiltrating lymphocytes: a prognostic factor of PSA-free survival in patients with local prostate carcinoma treated by radical prostatectomy. Anticancer Res. 2005;25:4435-8.

31. Fridman WH, Pages F, Sautes-Fridman C, et al. The immune contexture in human tumours: impact on clinical outcome. Nat Rev Cancer. 2012;12:298-306. https://doi.org/10.1038/nrc3245.

32. Jung K, Kang M, Park C, et al. Protective role of V-set and immunoglobulin domain-containing 4 expressed on kupffer cells during immune-mediated liver injury by inducing tolerance of liver Tand natural killer T-cells. Hepatology. 2012;56:1838-48. https:// doi.org/10.1002/hep.25906.

33. Tran PN, Sarkissian S, Chao J, et al. PD-1 and PD-L1 as emerging therapeutic targets in gastric cancer: current evidence. Gastrointest Cancer. 2017;7:1-11. https://doi.org/10.2147/GICTT.S113525.

34. Chen C, Zhang F, Zhou N, et al. Efficacy and safety of immune checkpoint inhibitors in advanced gastric or gastroesophageal junction cancer: a systematic review and meta-analysis. Oncoimmunology. 2019;8:e1581547. https://doi.org/10.1080/21624 02X.2019.1581547.

35. Cui J, Yin Y, Ma Q, et al. Comprehensive characterization of the genomic alterations in human gastric cancer. Int J Cancer. 2015;137:86-95. https://doi.org/10.1002/ijc.29352.
36. Charalampakis N, Economopoulou P, Kotsantis I, et al. Medical management of gastric cancer: a 2017 update. Cancer Med. 2018;7:123-33. https://doi.org/10.1002/cam4.1274.

37. Liu X, Meltzer SJ. Gastric cancer in the era of precision medicine. Cell Mol Gastroenterol Hepatol. 2017;3:348-58. https://doi. org/10.1016/j.jcmgh.2017.02.003.

38. Marshall HT, Djamgoz MBA. Immuno-oncology: emerging targets and combination therapies. Front Oncol. 2018;8:315. https:// doi.org/10.3389/fonc.2018.00315.

39. Goldstein J, Tran B, Ensor J, et al. Multicenter retrospective analysis of metastatic colorectal cancer (CRC) with high-level microsatellite instability (MSI-H). Ann Oncol. 2014;25:1032-8. https:// doi.org/10.1093/annonc/mdu100.

Publisher's Note Springer Nature remains neutral with regard to jurisdictional claims in published maps and institutional affiliations. 